\title{
Optimal oblique light illumination for photoacoustic microscopy beyond the diffusion limit
}

Christopher P. Favazza, Zijian Guo, Konstantin Maslov, Lihong V. Wang

Christopher P. Favazza, Zijian Guo, Konstantin Maslov, Lihong V. Wang, "Optimal oblique light illumination for photoacoustic microscopy beyond the diffusion limit," Proc. SPIE 7899, Photons Plus Ultrasound: Imaging and Sensing 2011, 789900 (28 February 2011); doi: 10.1117/12.874903 


\title{
Optimal oblique light illumination for photoacoustic microscopy beyond the diffusion limit
}

\author{
Christopher. P. Favazza ${ }^{a}$, Zijian Guo ${ }^{a}$, Konstantin Maslov ${ }^{\mathrm{a}}$, and Lihong V. Wang ${ }^{\text {ta }}$ \\ ${ }^{a}$ Optical Imaging Laboratory, Department of Biomedical Engineering, Washington University in St. \\ Louis, 1 Brookings Drive, St. Louis, MO, USA 63130;
}

\begin{abstract}
To image beyond the quasi-ballistic photon regime, photoacoustic tomography systems must rely on diffuse photons; however, there still exists an optimal illumination pattern that results in the largest number of photons reaching a target at a given depth. Many photoacoustic imaging systems incorporate weak optical focusing through oblique or dark-field illumination, but these systems are not often optimized for deep light delivery. Multiple parameters and constraints, particularly for in vivo imaging, need to be considered to determine the optimal illumination scheme for a given system: beam diameter, incident angle, pulse repetition rate, laser fluence, and target depth. Monte Carlo simulations of varied beam geometries and incident angles show the best optical illumination schemes for different imaging depths. Further an analytic model based on the diffusion theory provides a rapid method of determining the optimal beam size and incident angle for a given target depth and agrees well with the simulations. The results reveal the most efficient optical focal position to maximize the number of photons delivered to a target depth, therein maximizing the PA signal. The principles and results discussed here are not limited to the system investigated, but can be applied to other system configurations to improve the photoacoustic signal strength.
\end{abstract}

Keywords: photoacoustic microscopy, dark-field illumination, optimized fluence, oblique illumination

† lhwang@biomed.wustl.edu, http://oilab.seas.wustl.edu

\section{INTRODUCTION}

A major attribute of photoacoustic imaging is its superior depth penetration. The capability of super-depth imaging enables many potential clinical applications of photoacoustic tomography. Numerous photoacoustic imaging systems employed for human imaging experiments utilize oblique laser illumination to deliver photons to targets located in the diffusive optical regime [1-5]. Despite relying on diffuse photons for optical excitation of the photoacoustic signal, the illumination scheme can be optimized $[6,7]$ and some illumination schemes will generate greater target fluence than others. This is extremely important for clinical applications where safety is a major concern. By optimizing the laser illumination, the patient will be exposed to the minimum laser fluence necessary. Additionally, an increase in the signal to noise ratio generated by optimizing the fluence could reduce signal averaging or eliminate its necessity completely, should the application require it.

Here we show that the illumination scheme can be optimized. The optimization is predicated on several physical constraints: 1) At certain surface position relative to the ultrasound detection system, the incident angle of the laser is limited. 2) For patient safety, ANSI restricts the maximum permissible laser fluence. 3) The laser is limited in the pulse energy it can deliver to the target. We have applied these constraints and used the imaging parameters from out darkfield acoustic resolution photoacoustic microscope (AR-PAM) [8]. Additionally, only collimated beams were considered to promote broad illumination within the focal zone of the transducer. Although the results presented here are for our AR-PAM, the theoretical framework can be used to optimize other obliquely incident illumination schemes.

Photons Plus Ultrasound: Imaging and Sensing 2011, edited by Alexander A. Oraevsky, Lihong V. Wang, Proc. of SPIE Vol. 7899, 78990O - (C) 2011 SPIE · CCC code: 1605-7422/11/\$18 · doi: 10.1117/12.874903 


\section{METHODS}

\subsection{Physical constraints}

We constrained the illumination scheme with three different restrictions. The first limitations stems from the obstruction caused by the ultrasound transducer, which limits the available incident angles of the laser beam at certain positions on the surface of the sample. In general, the most efficient way to deliver photons to a target is to directly aim at the target, as depicted in Figure 1a. However, the presence of the transducer in the beam path precludes access to certain angles, and prevents direct aiming at the target, seen in Figlb. The best option is to use an angle that is as close to the direct aiming angle, shown pictorially in Figlc. The optimization formulation seeks to determine how efficient these illumination schemes are compared to directly aiming at the target but entering the sample much further away from the target.
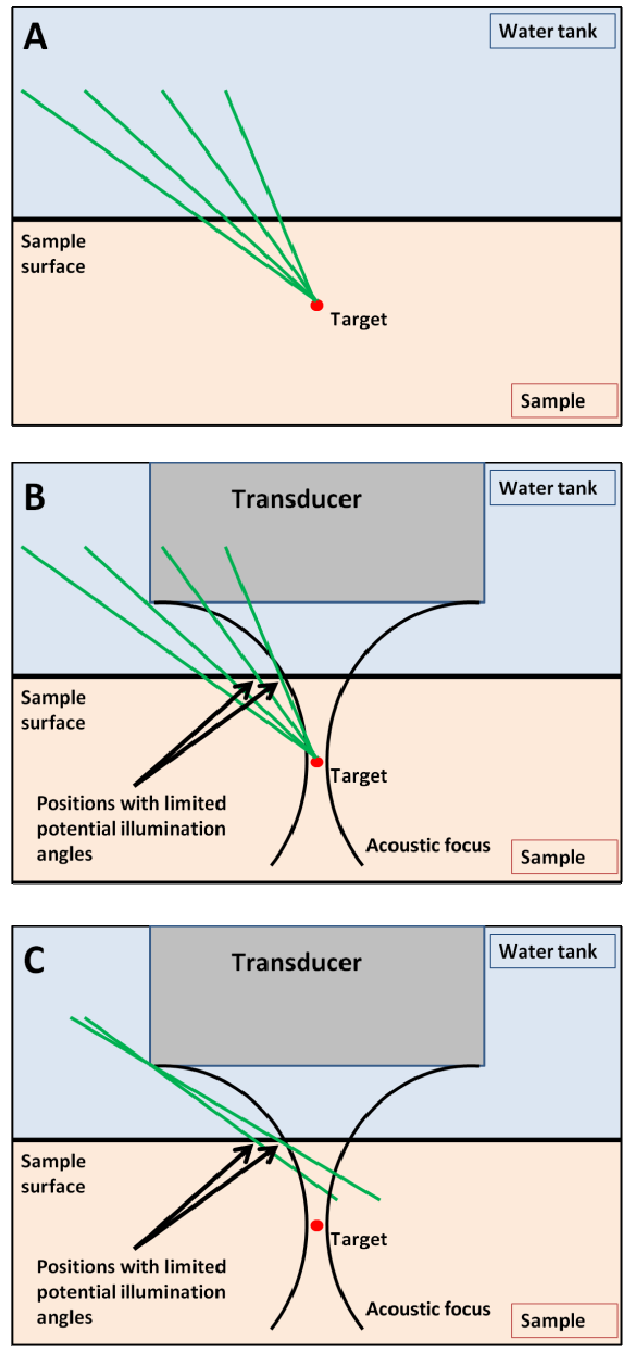

Figure 1. Cartoons describing the transducer obstruction restraint. (A) shows the optimal incident angle to illuminate a target for any position on the surface. (B) depicts the effect of the presence of the transducers with prevents direct aiming at certain surface positions. (C) shows the best available incident angle for restricted surface positions.

The second constraint is due to laser safety. ANSI limits laser exposure and restricts the permissible laser surface fluence to $20 \mathrm{~mJ} / \mathrm{cm}^{2}$. This value is further reduced if repetitive laser pulses are fired with a frequency in the $\mathrm{kHz}$ range. They enforce a correction factor of $\mathrm{n}^{-1 / 4}$, where $\mathrm{n}$ is the number of repetitive laser pulses. This is directly applicable to PAM as it is necessary to scan the surface of the sample to collect an image. The most number of consecutive pulses received by a given position on the sample's surface depends on the illumination pattern. For our AR-PAM, there are two possible 
surface illumination patterns, one where the geometric focus is above or beneath the surface (Figure 2a) or two, where the geometric focus is on the surface (Figure $2 b$ ). For the first case, the maximum number of consecutive laser pulses is determined by $2 \quad\left(r_{o}{ }^{2}-r_{i}^{2}\right)^{1 / 2}$ (step size), where $r_{o}$ and $r_{i}$ are the outer and inner radii of the illumination ring. In the case where the geometric focus resides on the sample, $\mathrm{n}$ can be calculated from the diameter of the overlapping beams, in which $\mathrm{n} \sim 2 \quad \mathrm{r}_{\mathrm{i}}$, or $\mathrm{n}$ can be calculated as $\mathrm{n} \sim 2 \quad\left(\mathrm{r}_{\mathrm{o}}{ }^{2}-\mathrm{r}_{\mathrm{i}}{ }^{2}\right)^{1 / 2}$. The first formulation is used if it more greatly reduces the energy density of the beam.

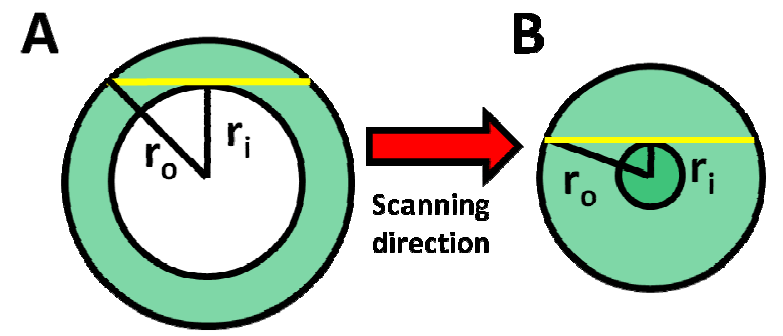

Figure 2. Two possible sample surface illumination patterns. (A) shows the pattern generated with the optical focus either above or beneath the surface. (B) depicts the pattern in which the focus is on the surface and the beams overlap. The yellow lines in (A) and (B) indicate the longest continuous path of the illumination pattern during scanning, from which the number of pulses is calculated for the correction factor. For certain cases where (B) represents the illumination pattern, the number of pulses will be calculated based the diameter of the inner circle for which the laser fluence is 4 times higher.

The third constraint is caused by a physical limitation of the laser source. Any laser will have a finite amount of pulse energy it can deliver. This is typically more of a significant factor in high repetition rate lasers, which are more beneficial for faster scanning systems. The second and third constraints are used to determine the optimal beam size and area for each illumination angle. The optimal beam size is defined as the minimum beam width $\left(r_{o}-r_{i}\right)$ that uses the maximum output of the laser and the maximum permissible laser fluence. We further use a collimated beam to maximize the fluence throughout the entire focal zone of the transducer. The optimal beam widths for various target depths and illumination angles are shown in Figure 3.
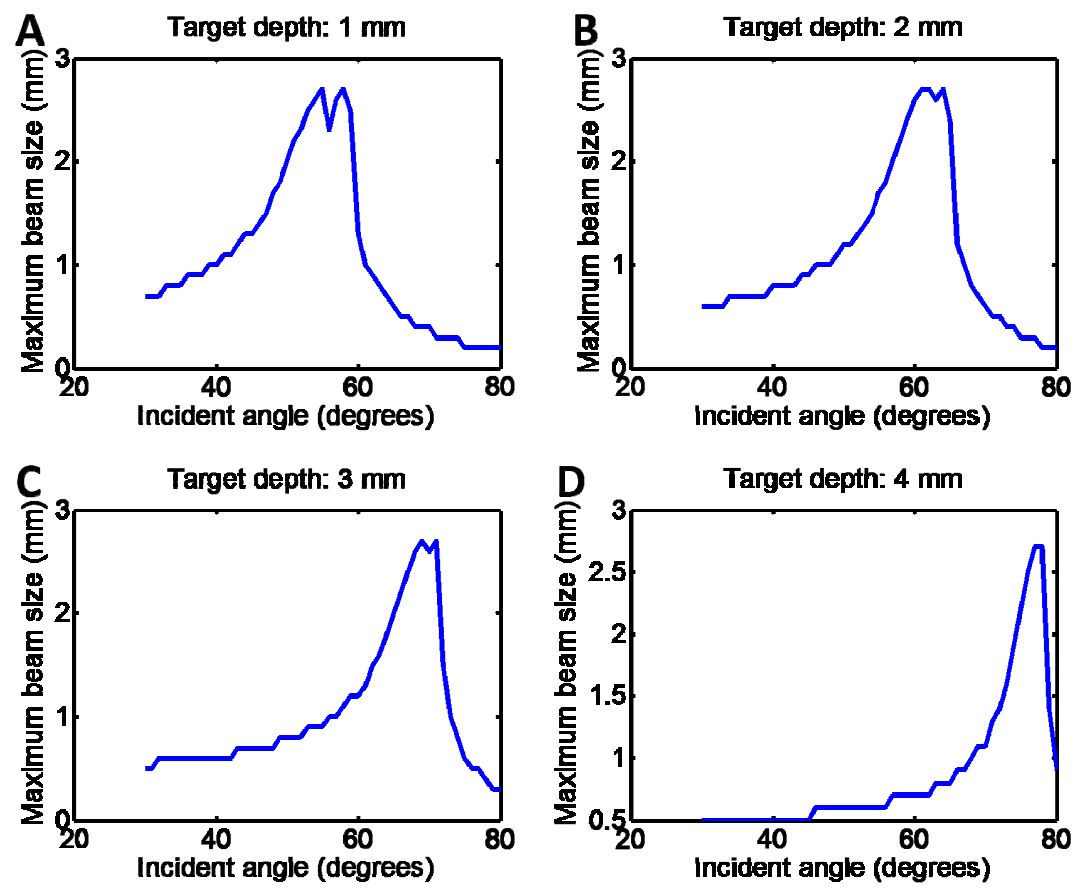

Figure 3. Optimal beam widths for illumination schemes with different incident angles. (A)-(D) show optimal beams widths for $1 \mathrm{~mm}, 2 \mathrm{~mm}, 3 \mathrm{~mm}$, and $4 \mathrm{~mm}$ target depths, respectively. 


\subsection{Monte Carlo model}

We employed the routinely used Monte Carlo photon transport model to simulate the different illumination angles [9]. More specifically, angled pencil beams consisting of 10 million photons were simulated for each angle and the three dimensional coordinates of each photon deposition event were recorded. The results were then convolved with different beam widths. The pencil beam results were convolved one dimensionally with the beam width because the results are spatially invariant in one dimension only. Due to rotational symmetry, the normalized convolution results were simply integrated over the area of the beam, and target fluence values were calculated. Sample Monte Carlo results for two illumination angles with different beam widths and varied target depths are shown in Figure 3. Clearly, for deeper targets, illumination schemes with larger incident angles will deliver more photons to the target than shallower angles.

\section{$70^{\circ}$ Incident angle}
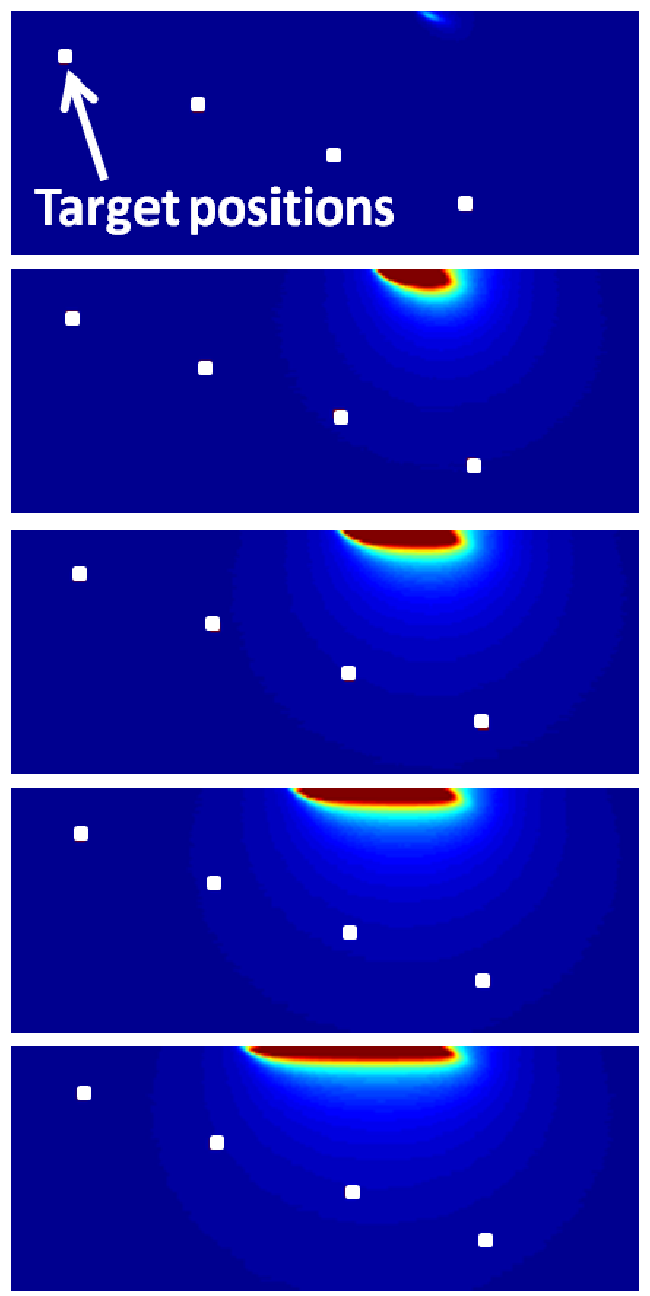

Pencil

\section{$1 \mathrm{~mm}$}

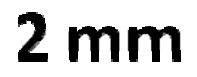

$3 \mathrm{~mm}$

$4 \mathrm{~mm}$
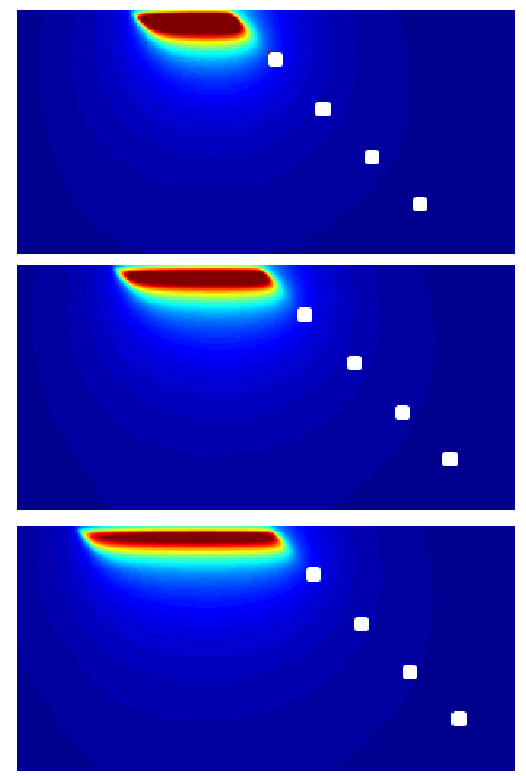

\section{$2 \mathrm{~mm}$}

Figure 4. Monte Carlo simulation results. The left panel shows simulation results for a $70^{\circ}$ incident angle. And the panel on the right shows the results for a $45^{\circ}$ incident angle. The target positions are indicated by the white boxes and are for $1 \mathrm{~mm}, 2$ $\mathrm{mm}, 3 \mathrm{~mm}$, and $4 \mathrm{~mm}$ depths. From the top image to the bottom image the beam width increases by $1 \mathrm{~mm}$ increments, with the top image depicting the pencil beam and bottom image showing a $4 \mathrm{~mm}$ wide beam. 


\subsection{Analytic model}

We developed an analytic model based on the diffusion approximation of the radiative transfer equation and compared it with the Monte Carlo model. The analytic model assumes that the beam entering the sample is a series of point sources. Each point source along the beam path is then weighted for scattering and absorption, which is determined by its position along the path and distance traveled in the turbid material. The shortest distance between the theoretical point source and target is then calculated and applied to the diffusion equation for a point source [10]. The contributions from all of the theoretical point sources are integrated over the entire beam width and path length, and the result is normalized to the total energy deposited. Hence the equation for the analytic model is:

$$
F_{\text {Norm }}=\frac{\iint \frac{\exp \left(\left(-\mu^{\prime} t^{*} l\right)+\left(\mu_{\left.\left.e f f^{*} p_{i}\right)\right)}\right.\right.}{4 \pi D p_{i}} d b d l}{\exp \left(\left(-\mu^{\prime} t^{*}\right) d b d l\right.},
$$

where $\mathrm{F}_{\text {Norm }}$ is the normalized target fluence, $\mu_{t}^{\prime}$ is the transport interaction coefficient, $D=\frac{1}{3\left[\mu_{a}+\mu^{\prime}{ }_{s}\right]}$ and is the diffusion coefficient $\left(\mu_{a}+\mu_{s}^{\prime}\right.$ are the absorption and reduced scattering coefficients), 1 is the distance between the point source and the sample surface, $\mathrm{p}_{\mathrm{i}}$ is the distance between the point source and the target, $\mu_{e f f}=\sqrt{\frac{\mu_{a}}{D}}$ is the effective attenuation coefficient, and $\mathrm{db}$ and $\mathrm{dl}$ represent infinitesimal increment in the beam width and path length respectively.

\section{RESULTS}

The optimal beam widths for various illumination angles and target depths were determined for a laser pulse energy of $0.7 \mathrm{~mJ}$. The optimal beam widths were then applied to both the Monte Carlo and analytic models. Both models reveal that it is more efficient to used larger incident angles and enter the surface under the transducer closer to the target, where direct aiming is impossible. Further, both models show that as the target depth increases, so does the optimal incident angle. Figure 5 shows these results and the good agreement between the Monte Carlo and simulation models.

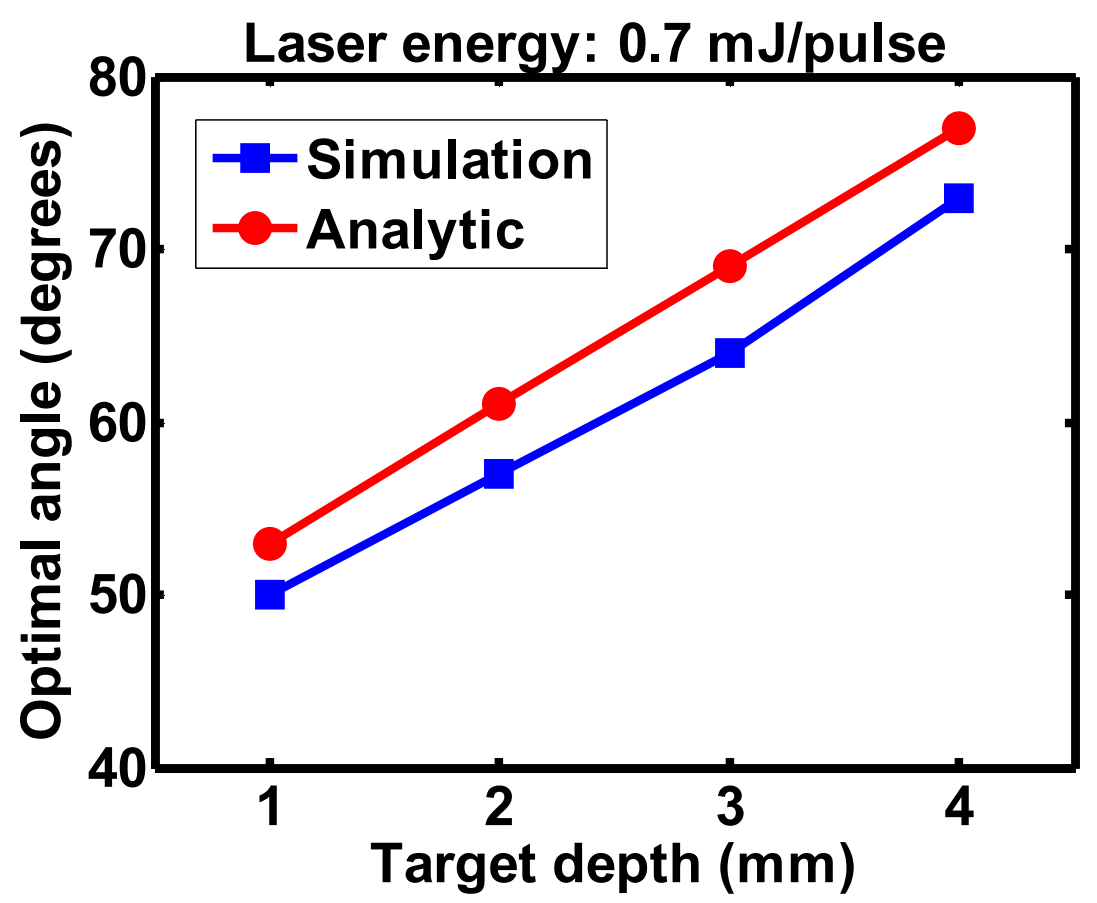

Figure 5. Comparative results from both the Monte Carlo and analytic models, which show the optimal illumination angles for different target depths. A $0.7 \mathrm{~mJ}$ maximum laser pulse was used in the optimization. 


\section{SUMMARY}

Although PAM relies on diffuse photon absorption for imaging deep targets, below the quasi-ballistic photon regime, there exist optimal illumination schemes that most efficiently deliver photons to the targets. Consequently, employing optimal schemes will result in higher signal to noise ratios, and/or reduces the necessary surface laser fluence. The results here were determined from applying the theoretical framework to our AR-PAM. However, the general framework is applicable to most any oblique illumination schemes. Since many photoacoustic imaging systems utilizes oblique

illumination, particularly those for direct clinical applications, these results could be beneficial to many different systems and applications.

\section{ACKNOWLEDGEMENTS}

This research was supported by National Institutes of Health grants R01 EB000712, R01 EB010049, R01 CA134539, R01 EB008085, R01 CA134539, U54 CA136398, and 5P60 DK02057933. LW acknowledges financial interest in Endra Inc., which, however, did not support this research.

\section{REFERENCES}

[1] H. F. Zhang, K. Maslov, G. Stoica et al., "Functional photoacoustic microscopy for high-resolution and noninvasive in vivo imaging," Nat Biotechnol, 24(7), 848-51 (2006).

[2] J. A. Viator, J. Komadina, L. O. Svaasand et al., "A comparative study of photoacoustic and reflectance methods for determination of epidermal melanin content," J Invest Dermatol, 122(6), 1432-9 (2004).

[3] L. Song, K. Maslov, K. K. Shung et al., "Ultrasound-array-based real-time photoacoustic microscopy of human pulsatile dynamics in vivo," J Biomed Opt, 15(2), 021303.

[4] R. G. Kolkman, P. J. Brands, W. Steenbergen et al., "Real-time in vivo photoacoustic and ultrasound imaging," J Biomed Opt, 13(5), 050510 (2008).

[5] C. Kim, T. N. Erpelding, L. Jankovic et al., "Deeply penetrating in vivo photoacoustic imaging using a clinical ultrasound array system," Biomed. Opt. Express, 1(1), 278-284.

[6] L. V. Wang, and G. Liang, "Absorption distribution of an optical beam focused into a turbid medium," Appl Opt, 38(22), 4951-8 (1999).

[7] L. V. Wang, R. E. Nordquist, and W. R. Chen, "Optimal beam size for light delivery to absorption-enhanced tumors buried in biological tissues and effect of multiple-beam delivery: a Monte Carlo study," Appl Opt, 36(31), 8286-91 (1997).

[8] K. Maslov, G. Stoica, and L. V. Wang, "In vivo dark-field reflection-mode photoacoustic microscopy," Opt Lett, 30(6), 625-7 (2005).

[9] L. Wang, S. L. Jacques, and L. Zheng, "MCML--Monte Carlo modeling of light transport in multi-layered tissues," Comput Methods Programs Biomed, 47(2), 131-46 (1995).

[10] L. V. Wang, and H.-I. Wu, [Biomedical optics : principles and imaging] Wiley-Interscience, Hoboken, N.J.(2007). 